\title{
évolution de l'adhérence des argiles sur des surfaces métalliques en fonction du temps
}

\author{
par \\ J. Y. Boisson \\ et \\ J. P. Longuemard
}

\section{Introduction}

L'activité de l'homme au fond des mers s'intensifie et s'oriente notamment vers les domaines de l'exploitation de ressources minières présentes sur certains grands fonds océaniques. En conséquence, l'obligation de soulever, déplacer ou faire circuler des objets ou des véhicules en contact direct avec les argiles marines nécessite de mieux connaître les modalités de résistance par phénomène d'adhérence qui prend naissance entre un sédiment cohésif faiblement consolidé et une surface métallique.

Le travail présenté ici prend place dans le cadre d'un vaste projet d'étude de chantier sous-marin destiné au ramassage des nodules polymétalliques. L'exploitation de ces nodules sera effectuée par navettes autonomes propulsées par vis d'Archimède en appui sur des sédiments argileux.

Nous nous proposons d'étudier spécialement le rôle du temps de contact sur l'évolution du phénomène d'adhérence à l'origine d'éventuelles pertes de rendement dans le fonctionnement des navettes, en particulier lors d'une remise en mouvement après un arrêt plus ou moins prolongé.

Les temps de collage maximum retenus pour ces essajs sont de l'ordre de 24 heures. En effet, un engin immobilisé sur un fond argileux pendant une durée supérieure à cette limite a subi une avarie de fonctionnement telle qu'elle n'autorise plus l'espoir de la remettre en mouvement.

Les travaux ont été conduits en laboratoire pour apprécier le phénomène. Une campagne d'essais in situ, en milieu marin, permet de vérifier nos conclusions et de proposer un modèle mathématique.

\section{Généralités sur l'adhérence}

L'adhérence est la résistance au cisaillement nécessaire pour rompre le collage qui lie la surface d'un objet à un sédiment cohérent.
Le phénomène de l'adhérence se rencontre fréquemment pour de nombreux problèmes de mécanique des sols. Les principaux domaines où l'on doit prendre en compte ce paramètre sont la pénétrométrie, les modélisations de calculs. de pieux notamment à l'arrachage, l'étude de tenue de différents systèmes d'ancrage sous-marins.

Le frottement latéral unitaire que l'on rencontre dans le cas de sol cohérent représente en fait l'adhérence de cette argile ou, au maximum, sa cohésion (Tomlinson [14]).

Le principal point étudié par les auteurs (Tomlinson $[13,14]$, Caquot et Kerisel [2]) est le paramètre $\alpha$ ou facteur d'adhérence qui est le rapport entre l'adhérence (ou frottement latéral unitaire en milieu cohérent) et la cohésion non drainée du sol. La synthèse de nombreuses mesures effectuées sur des essais d'arrachement de pieux constitués par différents matériaux fait apparaître que la cohésion non drainée du sol considéré est un facteur significatif. On observe une nette diminution du facteur d'adhérence $\alpha$ pour les cohésions croissantes de 500 à $3000 \mathrm{~g} / \mathrm{cm}^{2}$.

La figure 1 permet d'apprécier le rôle relatif du temps de collage préalable $t$ sur l'adhérence $\tau_{\mathrm{B}}$ dans le cas d'une plaque testée sur une vase naturelle d'origine marine provenant de la baie de Banyulśs (PyrénéesOrientales) dont les caractéristiques géotechniques au moment des essais sont résumées en annexe.

A temps de collage nul $(t=0)$ l'adhérence en fonction de la contrainte $\sigma_{n}$ suit une loi quasi linéaire de type :

$$
\tau_{\mathrm{a}}=\mathrm{a}+\sigma_{\mathrm{n}} \operatorname{tg} \delta
$$

où $\delta$ (angle de frottement sédiment-plaque) est pratiquement nul et $\tau_{\mathrm{a}}$ (adhérence à l'origine des contraintes) est de très faible valeur.

L'accroissement de l'adhérence $\tau_{a}$ en fonction du temps suit une loi similaire pour chaque contrainte utilisée. Au-delà d'un temps de collage supérieur à une dizaine d'heures (fig. 2), l'adhérence tend vers une limite qui augmente avec la contrainte $\sigma_{n}$. Elle atteint 


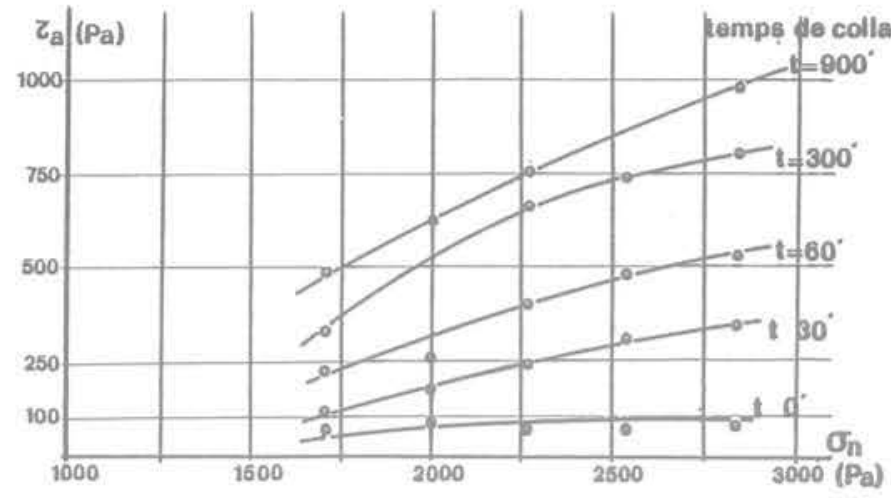

Fig. 1 Loi $\tau=f\left(\sigma_{n}\right)$ à différents temps de collage pour une plaque sur sédiment de la baie de Banyuls

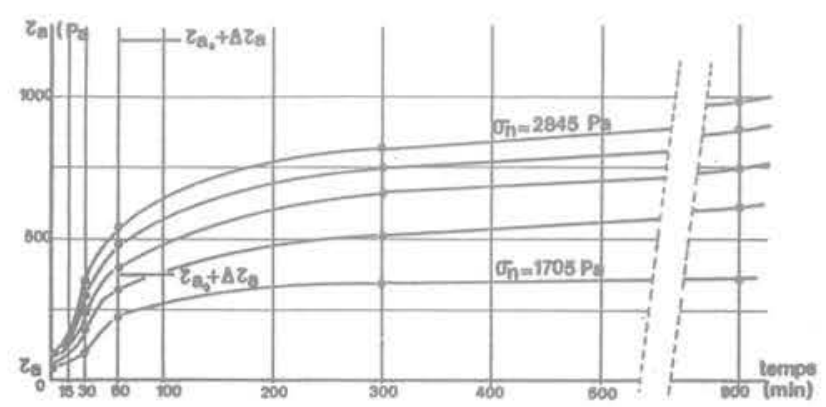

Fig. 2 Loi $\tau_{a}=f(t)$ pour une plaque de sédiment de la baie de Banyuls

une valeur proche de celle de la cohésion $c_{u}$ pour la contrainte la plus élevée.

II est donc possible de préciser la loi $\tau_{\mathrm{a}}=\mathrm{f}(\mathrm{t})$ pour ce sédiment. Nous pouvons reconnaître :

- l'existence d'une valeur minima initiale $\approx \tau_{a_{0}}$ " qui varie en fonction de la contrainte $\sigma_{n}$ induite selon une loi du type:

$$
\tau_{\mathrm{a}_{0}}=\mathrm{a}+\sigma_{\mathrm{n}} \operatorname{tg} \delta
$$

- un temps de collage noté to pour lequel $\tau_{\mathrm{a}_{0}}$ garde une valeur constante, $t_{0}$ est pratiquement inexistant pour les plus fortes contraintes;

- une rapide progression des valeurs de l'adhérence pour un temps de collage $t$ supérieur à $t_{0}$. Cette progression est d'autant plus rapide que le contact entre le sédiment et la plaque est intense (augmentation de la contrainte $\sigma_{n}$ );

- l'existence d'une valeur limite d'adhérence pour un temps de collage préalable d'une dizaine d'heures minimum. Cette valeur limite se rapproche de la valeur de la cohésion $c_{u}$ mesurée sur le sédiment pour les pressions induites par la plaque $\left(\sigma_{n}\right)$ les plus élevées.

L'adhérence $\tau_{\mathrm{a}}$, en fonction du temps de collage préalable entre plaque d'acier et sédiment argileux, peut s'exprimer sous la forme générale de l'équation :

$$
\tau_{\mathrm{a}}=\tau_{\mathrm{a}_{0}}+\Delta \tau_{\mathrm{a}}\left[1-\exp -\left[\left(\mathrm{t}-\mathrm{t}_{\mathrm{o}}\right) / \lambda\right] \text { pour } \mathrm{t}>\mathrm{t}_{\mathrm{o}}\right.
$$

et

$$
\tau_{\mathrm{a}}=\tau_{\mathrm{a}_{0}} \text { pour } \mathrm{t}<\mathrm{t}_{\mathrm{o}}
$$

$\tau_{\mathrm{a}}=$ adhérence entre plaque et sédiment (exprimée en $\mathrm{Pa}$ )

$\tau_{\mathrm{a}_{0}}=$ adhérence initiale existant pour un temps de collage nul et constante pendant un temps $t_{0}$ $\tau_{a_{0}}$ suit une loi de type: $\tau_{a_{0}}=\sigma_{n} \operatorname{tg} \delta$

$\Delta \tau_{\mathrm{a}}=$ valeur d'adhérence telle que $\tau_{\mathrm{B}_{0}}+\Delta \tau_{\mathrm{a}}=\tau_{\mathrm{a}}$ (limite de $\left(\tau_{a_{0}}+\Delta \tau_{a}\right)$ proche de la valeur $c_{u}$ pour les plus fortes contraintes)

$t_{0}=$ temps pendant lequel l'adhérence $\alpha \tau_{\mathrm{a}}$ " garde une valeur $\tau_{\mathrm{B}_{0}}$ constante (exprimé en minutes)

$t=$ temps de collage entre surface et sédiment avant traction

$\lambda=$ constante de temps exprimée en minutes.

Les valeurs moyennes de ces différents paramètres calculés à partir des valeurs d'adhérence mesurées avec une plaque en acier brut d'usinage tractée à faible vitesse sur le sédiment de la baie de Banuyls, sont les suivantes :

- pour la plus faible contrainte $\sigma_{n}=1717 \mathrm{~Pa}$

$$
\tau_{\mathrm{B}}=50+350[1-\exp -[(\mathrm{t}-20) / 65]]
$$

La somme $\tau_{\mathrm{a}_{0}}+\Delta \tau_{\mathrm{a}}=400 \mathrm{~Pa}$ représente la valeur asymptotique d'adhérence pratiquement atteinte après 1000 minutes $(16 \mathrm{~h})$ de collage préalable.

- pour la plus forte contrainte $\sigma_{n}=2845 \mathrm{~Pa}$

$$
\tau_{\mathrm{a}}=100+900[1-\exp -(\mathrm{t}-5) / 60]
$$

Les variations $\tau_{\mathrm{a}}$ (de 50 à $100 \mathrm{~Pa}$ ) représentent en fait la variation simple de l'adhérence en fonction de la contrainte déterminée au § 3.2 .1 suivant la loi :

$$
\tau_{\mathrm{a}_{0}}=\mathrm{a}+\sigma_{\mathrm{n}} \operatorname{tg} \delta
$$

Les trois facteurs qui varient de manière significative vis-à-vis des influences du temps et de la contrainte sont : $\Delta \tau_{\mathrm{a}}, t_{0}$ et $\lambda$.

- $t_{0}$ (temps jusqu'auquel il n'y a pas de variations de

l'adhérence en fonction du temps : $\tau_{a}=\tau_{a_{0}}=$ Cte)

diminue avec les contraintes croissantes: de $20 \mathrm{mn}$ pour $\sigma_{n}=1717 \mathrm{~Pa}$ à $5 \mathrm{mn}$ pour $\sigma_{\mathrm{n}}=$ $2845 \mathrm{~Pa}$;

- $\Delta \tau_{\mathrm{a}}$ (valeur telle que $\tau_{\mathrm{a}_{0}}+\Delta \tau_{\mathrm{a}}=$ Cte pour un temps très long (une journée) augmente très sensiblement avec la contrainte jusqu'à ce que $\tau_{\mathrm{a}_{0}}+\Delta \tau_{\mathrm{a}} \approx \mathrm{c}_{\mathrm{u}}$ pour les plus fortes contraintes, $\Delta \tau_{a}$ croît de $450 \mathrm{~Pa}$ à $900 \mathrm{~Pa}$

- $\lambda$ (constante de temps, caractéristique de la rapidité d'augmentation de l'adhérence en fonction du temps pour $t$ supérieur à $t_{0}$ ).

Afin de confirmer cette loi $\tau_{a}=f(t)$ sur d'autres types d'argiles et de reconnaitre ainsi les variations qui peuvent affecter les principaux paramètres de cette loi, nous avons repris ces essais sur trois autres sédiments reconstitués, aussi différents que possible les uns des autres. Chaque série a été réalisée dans des conditions d'expérimentation strictement semblables (plaque, vitesse, contraintes et temps de collage).

Les sédiments utilisés respectivement pour chacune de ces séries sont :

- de la vase de l'étang de Salses-Leucate,

- une argile pure (kaolinite),

- un mélange vase de la baie de Banyuls et $25 \%$ de bentonite.

Leurs caractéristiques physiques au moment des essais sont précisées en Annexe $n^{\circ} 1$.

Meyerhof [9] évalue ce rapport $\alpha$ entre 0,1 et 0,3 pour des plaques en acier poli en contact avec des argiles dans le cas d'une modélisation en laboratoire des forces nécessaires à l'extraction de systèmes d'ancrage enfouis dans un sédiment.

Witney (1968) [17] donne une valeur moyenne de $\alpha$ de $11 \%$ dans le cas du contact verre/argile (cohésion de l'argile $=16,6 \mathrm{~g} / \mathrm{cm}^{2}$ ). 
Erchul et Smith (1969) [5] ont estimé la valeur de l'adhérence à $25 \%$ de la cohésion.

Plus récemment, toujours en étudiant la résistance à l'extraction de systèmes d'ancrage enfouis dans des sédiments marins, Wang-Demars et Nacci (1977) [16] chiffrent le rapport adhérence/cohésion de $67 \%$ à $75 \%$.

II ressort donc nettement de ces études que l'adhérence d'un sédiment sur une surface quelconque est fonction de la cohésion. Cette notion ne peut se développer dans le cas de sables où le frottement intervient seul.

De la même manière Chari, Guha et Mutukrishaian (1979) [4] ont montré que l'adhérence était également fonction de l'état de surface de l'objet considéré et de la cohésion du sédiment sans qu'il y ait dans tous les cas une relation proportionnelle directe.

Notons que, pour ces essais en laboratoire, les plus grandes cohésions sont atteintes après un temps de repos de l'argile d'environ $72 \mathrm{~h}$, donc un temps de collage équivalent pour les objets testés. Les valeurs d'adhérence les plus faibles sont mesurées à un temps de collage plaque-sédiment de l'ordre de deux heures. Cela laisse supposer que le temps de contact influence aussi l'évolution des valeurs d'adhérence mesurées.

Vesic (1971) [15] énonce l'hypothèse que l'adhérence peut être égale à la cohésion au-delà d'une période allant de quelques jours à plusieurs mois.

En fait, tout sédiment cohérent se compose de particules argileuses dont l' "activité " développe un processus d'adhérence au contact avec une surface quelle qu'en soit la nature. Ce processus physicochimique se développe avec le temps. L'accroissement de l'adhérence serait lié au phénomène de régénération de la cohésion détruite ou modifiée par le contact objet-sédiment.

\section{Analyse expérimentale}

Les mesures en nature ont l'avantage de dépendre de la résultante de tous les facteurs susceptibles d'intervenir localement. Elles sont donc utilisables dans des calculs de modélisation d'engins en contact avec des fonds marins. Cependant, les aléas des manipulations et des conditions atmosphériques en mer rendent ces essais complexes et longs. Ils limitent de ce fait le nombre et le choix des sites. II nous a paru plus logique d'aborder la présente étude par des essais en laboratoire sur des sédiments reconstitués. Des expériences, effectuées in situ, ont ensuite permis de vérifier les conclusions des données acquises en laboratoire.

\subsection{Essais en laboratoire}

L'étude expérimentale a été menée à l'aide de plaques d'acier posées sur quatre types d'argiles de nature minéralogique aussi différentes que possible. Cependant, l'état physique de ces sédiments reconstitués (mesuré au moment des essais, cf. Annexe 1) est relativement proche de celui d'une argile marine en place: faible cohésion, faible consolidation.

L'état de surface retenu pour la plaque testée est celui d'un acier brut d'usinage rouillé.

II correspond à l'éventualité la plus pessimiste en ce qui concerne le matériau qui pourrait constituer les vis destinées à la propulsion des engins de ramassage de nodules polymétalliques.

L'adhérence entre la surface métallique et le sédiment, fonction de la contrainte imposée par la plaque, dépend pro-parté du temps de collage.

L'évolution des adhérences mesurées en fonction du temps de collage préalable suit une loi identique à celle proposée précédemment pour chacun des sédiments testés.

L'ensemble des résultats obtenus à partir de ces essais de laboratoire est résumé dans le tableau $n^{\circ} 1$. Les valeurs caractérisant la fonction $\tau_{\mathrm{a}}=\mathrm{f}(\mathrm{t})$ (tabl. 1) sont extraites d'un nombre important de mesures d'effort de traction. Chacune d'entre elles est obtenue en moyennant les résultats provenant d'une dizaine d'essais. Les valeurs des différents paramètres de la loi $\tau_{a}=f(t)$ sont ainsi comparées, pour deux contraintes $\sigma_{n}=1717 \mathrm{~Pa}$ et $\sigma_{n}=2845 \mathrm{~Pa}$, entre les quatre sédiments testés. Les paramètres géotechniques retenus pour caractériser chacun de ces quatre sédiments sont la cohésion $c_{u}$ mesurée au Fall Cône Test, la teneur en eau et l'indice de liquidité $\mathrm{L}_{\mathrm{L}}$.

Ces valeurs précisent le comportement spécifique de l'adhérence en fonction du temps pour chacune des argiles vis-à-vis de la plaque :

- sédiment de Salses-Leucate

- de la valeur des différents paramètres nous constatons que l'évolution de l'adhérence de ce sédiment est assez semblable à celle du sédiment de la baie de Banyuls;

- pour la plus forte contrainte $\sigma_{n}$ testée, la somme de $\tau_{\mathrm{a}_{0}}+\Delta \tau_{\mathrm{a}}=1000 \mathrm{~Pa}$ au bout d'une vingtaine d'heures de collage est pratiquement égale à la cohésion $c_{u}$.

- kaolinite pure

- les valeurs d'adhérence mesurées, même à forte contrainte et long temps de collage, sont proportionnellement faibles par rapport aux valeurs moyennes de

Tableau $n^{\circ} 1$

Comparaison entre les paramètres de la loi $\tau_{\mathrm{a}}=\mathrm{f}(\mathrm{t})$ pour quatre argiles testées en laboratoire

\begin{tabular}{|c|c|c|c|c|c|c|c|c|c|c|c|c|c|c|c|c|}
\hline \multirow[b]{2}{*}{ Sédiment testé } & \multirow[b]{2}{*}{ WI } & \multirow[b]{2}{*}{$\begin{array}{l}\mathrm{c}_{u} \\
(\mathrm{~Pa})\end{array}$} & \multirow[b]{2}{*}{ Ip } & \multirow[b]{2}{*}{$I_{L}$} & \multicolumn{6}{|c|}{$\sigma_{n}=1717 \mathrm{~Pa}$} & \multicolumn{6}{|c|}{$\sigma_{n}=2845 \mathrm{~Pa}$} \\
\hline & & & & & $\begin{array}{l}7=0 \\
(\mathrm{~Pa})\end{array}$ & $\begin{array}{l}\Delta \mathrm{T}_{0} \\
(\mathrm{~Pa})\end{array}$ & $\begin{array}{c}\mathrm{t}_{0} \\
(\mathrm{~min})\end{array}$ & $\frac{\lambda}{(\mathrm{min})}$ & $\alpha=\frac{T_{\mathrm{a} 0}}{C_{u}}$ & $a^{\prime}=\frac{\tau_{m a}+\Delta \tau_{a}}{c_{u}}$ & $\begin{array}{l}\tau_{a 00} \\
(\mathrm{~Pa})\end{array}$ & $\begin{array}{l}\Delta \tau_{a} \\
(\mathrm{~Pa})\end{array}$ & $\begin{array}{c}10 \\
(m i n)\end{array}$ & $\begin{array}{c}\lambda \\
(\min )\end{array}$ & $\alpha=\frac{\tau_{a 0}}{c_{u}}$ & $\alpha^{\prime}=\frac{\tau_{a 0}+\Delta \tau_{a}}{c_{u}}$ \\
\hline $\begin{array}{l}\text { Baie de } \\
\text { Banyuls }\end{array}$ & 48,4 & 1200 & 8.7 & 2,3 & 50 & 350 & 20 & 65 & 0,04 & 0,42 & 100 & 900 & 5 & 100 & 0.06 & 0,83 \\
\hline $\begin{array}{l}\text { Etang de } \\
\text { Salses-Leucate }\end{array}$ & 77,5 & 1000 & 13 & 3,1 & 60 & 300 & 15 & -62 & 0.04 & 0.34 & 100 & 820 & 10 & -60 & 0.18 & 1 \\
\hline Kaolinite & 42 & 5000 & 19 & 0,8 & 100 & 250 & $\sim 0$ & -75 & 0.02 & 0.07 & 250 & 550 & -0 & -35 & 0,05 & 0,16 \\
\hline $\begin{array}{l}\text { Mélange } \\
75 \% \text { Banyuls } \\
25 \% \text { bentonite }\end{array}$ & 68 & 700 & 8.9 & 4,4 & 50 & 450 & 30 & -173 & 0.07 & 0.71 & 100 & 900 & 25 & $\sim 100$ & 0.14 & $\begin{array}{c}>1 \\
(1,43)\end{array}$ \\
\hline
\end{tabular}


cohésion apparentes de ce sédiment:

- il n'y a pratiquement pas de temps de collage $t_{0}$ pendant lequel l'adhérence $\tau_{\mathrm{a}}$ garde une valeur minima constante;

- si l'évolution de cette adhérence en fonction du temps peut encore s'assimiler à une loi de la forme $\tau_{\mathrm{a}}=\tau_{\mathrm{a}_{0}}+\Delta \tau_{\mathrm{a}}(1-\exp -\mathrm{t} / \lambda)\left(\mathrm{Nb}: \mathrm{t}_{\mathrm{o}} \sim 0\right)$ il y a augmentation relativement faible de $\tau_{a}$ par rapport à $\tau_{a_{0}}$ :

- pour $\sigma_{n}=1717 \mathrm{~Pa}: \tau_{\mathrm{a}} \operatorname{maxi}=3,5 \tau_{\mathrm{a}_{0}}$

- pour $\sigma_{n}=2845 \mathrm{~Pa}: \tau_{\mathrm{a}} \operatorname{maxi}=3,2 \tau_{\mathrm{a}_{0}}$

Ces résultats signifient que l'augmentation de $\tau_{\mathrm{a}}$ en fonction du temps se fait dans les mêmes proportions, quelle que soit la contrainte induite par la plaque sur le sédiment.

De par sa structure ionique, la kaolinite est un minéral peu actif d'un point de vue électrique. De plus, en raison de la taille, relativement importante des particules qui le constituent, le sédiment est peu susceptible de modifications dans sa structure lorsqu'il est soumis à des sollicitations physiques externes. II présente une faible sensibilité à l'eau.

L'ensemble de ces facteurs permet d'expliquer et de confirmer à la fois :

- les faibles valeurs du rapport adhérence/cohésion.

- la faible influence du temps sur cette adhérence dans le cas du collage entre une plaque métallique et la kaolinite pure.

- Mélange : $75 \%$ vase de la baie de Banyuls $25 \%$ bentonite

- après un temps de collage initial $t_{0}$ compris entre $25 \mathrm{~min}$ et $30 \mathrm{~min}$ pour lequel les valeurs d'adhérence varient dans de très faibles proportions, le mélange testé montre une assez forte sensibilité de l'adhérence vis-à-vis du temps :
- les valeurs de $\tau_{\mathrm{a}_{0}}$ pour les deux contraintes étudiées sont identiques à celles mesurées pour les essais sur des sédiments de Banyuls seuls. Cependant, compte tenu de la cohésion $\mathrm{c}_{u} \sim 700 \mathrm{~Pa}$, cette adhérence est relativement plus importante. Pour ce mélange, la valeur de $\alpha$ définie comme le rapport $\tau_{\mathrm{a}_{0}} / \mathrm{c}_{\mathrm{u}}$ varie de 0,07 à 0,14 . Ce rapport variait de 0,04 à 018 dans le cas du sédiment de la baie de Banyuls;

- les plus longs temps de collage utilisés (900 min) permettent un accroissement encore notable de $\tau_{\mathrm{a}}$ bien que dans le cas de la plus forte contrainte $\left(\sigma_{n}=\right.$ $2845 \mathrm{~Pa}$ ) la valeur de $\tau_{\mathrm{a}}$ (à $\mathrm{t}=900 \mathrm{~min}$ ) soit proche de la cohésion $c_{u}$ mesurée au F.C.T. sur ce sédiment.

II est évident que la nature minéralogique de l'argile testée est une des raisons majeures pouvant expliquer les variations de comportement au collage d'une plaque sur un sédiment en fonction du temps.

Les différences de sensibilité d'une argile à l'adhérence vis-à-vis du temps de collage préalable sont illustrées par l'étude comparée, pour chaque sédiment, du rapport $\alpha=\tau_{\mathrm{a}_{0}} / \mathrm{c}_{\mathrm{u}}$ ou $\alpha^{\prime}=\left(\tau_{\mathrm{a}_{0}}+\Delta \tau_{\mathrm{a}}\right) / \mathrm{c}_{\mathrm{u}}$ aux deux contraintes $\sigma_{n}$ extrêmes utilisées. Les deux temps retenus sont $t=0$ et $t>24 \mathrm{~h}$ (fig. 3 ). Cette étude montre que :

- l'adhérence est, par rapport à la cohésion, plus faible et moins sensible dans le cas de la kaolinite;

- l'adhérence est nettement plus importante et plus susceptible de varier dans le cas des deux sédiments d'origine marine;

- la bentonite accroît de manière sensible l'importance de $\tau_{\mathrm{a}}$. Dans ce cas, plus que dans tout autre, la conjugaison des deux facteurs (forte contraintelong temps de collage) a pour effet d'accentuer le phénomène.

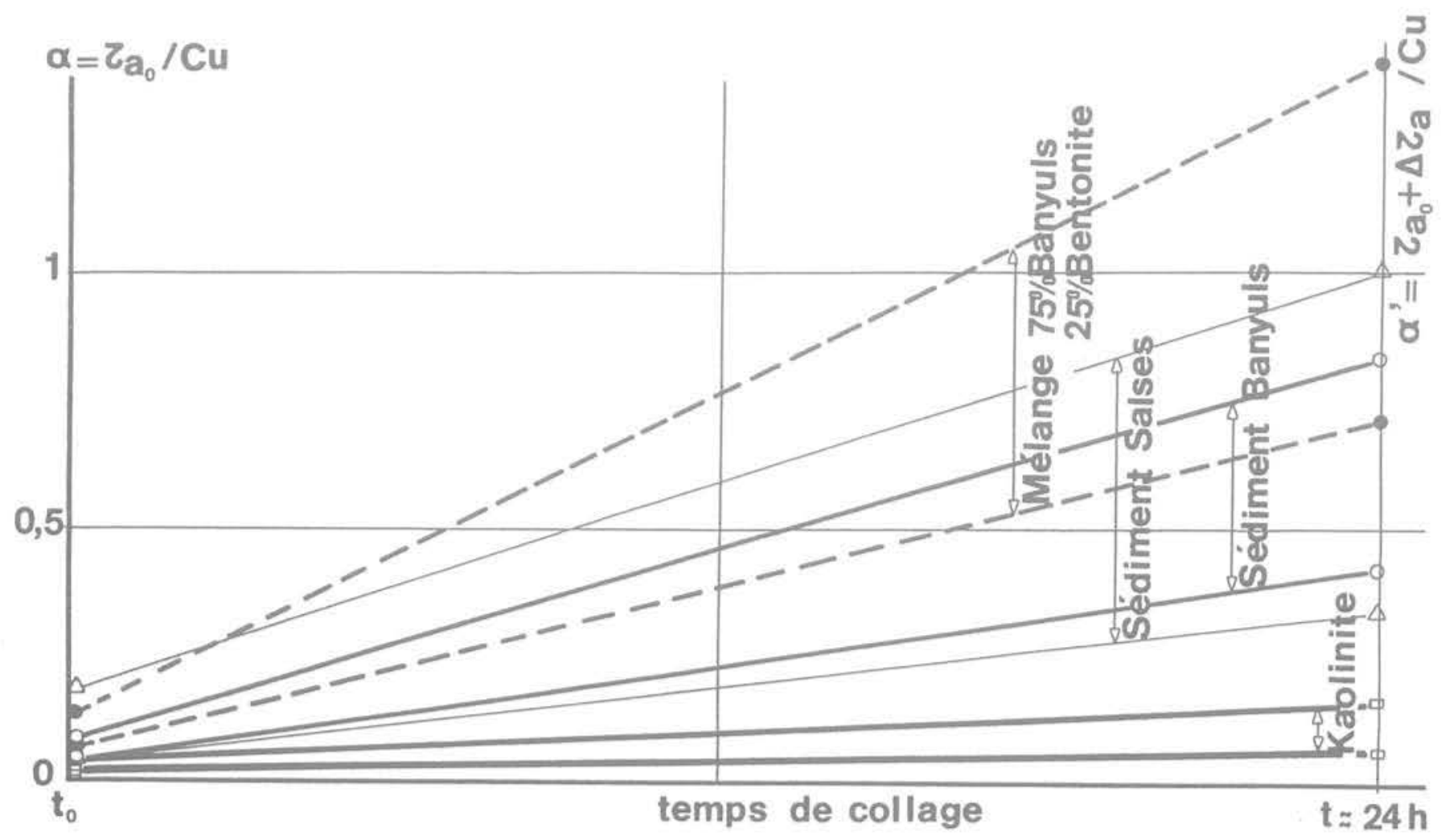

Fig. 3 Rapports $\alpha$ et $\alpha^{\prime}$ en fonction du temps pour une plaque sur quatre sédiments argileux 
On notera également, sans tenir compte de la minéralogie, que les remarques précédentes peuvent être formulées de manière identique si l'on se réfère uniquement à l'état physique du sédiment.

En effet, la kaolinite est l'argile qui présentait la plus faible teneur en eau (W\% 42\%) et le plus faible indice de liquidité $\left(I_{L}=0,78\right)$.

A l'inverse, le mélange $75 \%$ sédiment de la baie de Banyuls $-25 \%$ bentonite possède une teneur en eau $W \%$ de $68 \%$ et un indice de liquidité $I_{L}$ de 3,4 .

\subsection{Essais in situ}

Une étude de ladhérence $\tau_{\mathrm{a}}$ sur fonds marins a été entreprise à l'aide de plaques métalliques enfoncées dans le sédiment par gravité puis retirées après différents temps de contact.

Le principe de ces manipulations et la description du dispositif expérimental utilisé sont présentés dans l'Annexe 2.

Les résultats de cette campagne d'essais permettent de confirmer la modélisation mathématique sur l'évolution de $\tau_{a}$ en fonction du temps proposé à partir des essais en laboratoire. Ils permettent également de préciser les différences constatées pour un sédiment de même origine entre essais in vitro et essais en nature.

Deux sites marins ont été retenus pour ces manipulations :

- un sédiment peu profond ( -4 mètres) dans l'étang de Salses-Leucate (Pyrénées-Orientales) au point noté ES 5;

- un fond argileux ( -30 mètres) dans la baie de Banyuls (Pyrénées-Orientales) aux points notés B 15 et B 21.

Les caractéristiques sédimentologiques et géotechniques de ces deux formations ont été déterminées après prélèvement d'échantillons (cf. Annexe 2).

Pour chaque essai on détermine les paramètres suivants :

- une adhérence initiale $\tau_{\mathrm{a}_{0}}$ correspondant à la force de collage unitaire due au sédiment. Elle est définie comme la force s'opposant à l'enfoncement de la plaque au bout de quelques instants après la mise en place:

- une adhérence $\tau_{a}$ mesurée après un temps de collage préalable $t$. Elle est définie comme la force de collage unitaire s'opposant à l'arrachage de la partie de la plaque enfouie dans le sédiment.

L'évolution de l'adhérence $\tau_{a}$ en fonction du temps pour les essais effectués à l'étang de Salses-Leucate (point ES 5) est fournie sur la figure 4.

Malgré une différence parfois sensible quant aux valeurs de $\tau_{\mathrm{g}}$ trouvées pour chacune des quatre surfaces de plaques - différences qui peuvent être imputées aux difficultés de manipulations inhérentes aux essais en mer et aux erreurs qu'elles entraînent -. l'ensemble des valeurs d'adhérence en fonction du temps de collage montre une évolution caractéristique tout à fait comparable aux lois déterminées en laboratoire sur différentes argiles.

Les données acquises concernent des plaques de surfaces comprises entre 0,25 et $2 \mathrm{~m}^{3}$. Elles confirment l'indépendance totale de $\tau_{\mathrm{a}}$ en fonction de la surface de contact.

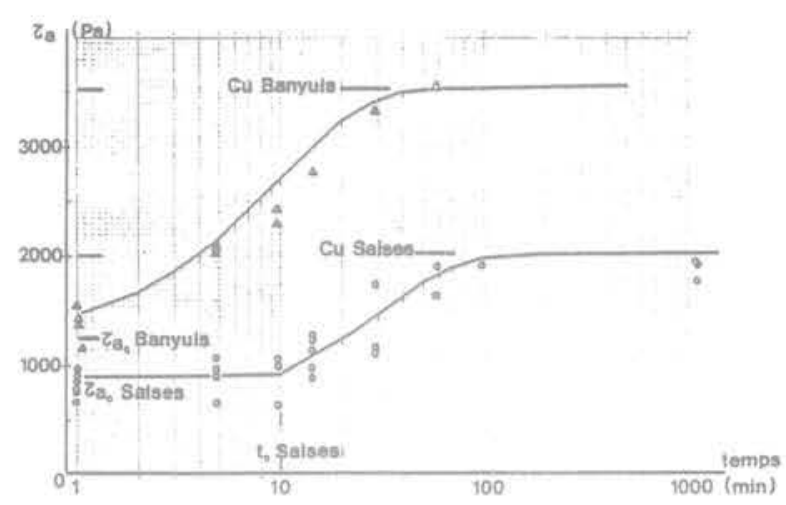

Fig. 4 Loi $\tau_{2}=f(t)$ pour essais in situ sur sédiments de l'étang de Salses-Leucate et de la baie de Banyuls

Rappelons que cette loi $\tau_{\mathrm{a}}=\mathrm{f}(\mathrm{t})$ s'exprime comme suit : $\tau_{\mathrm{B}}=\tau_{\mathrm{B}_{0}}+\Delta \tau_{\mathrm{a}}\left(1-\exp \left[\left(t-t_{0}\right) / \lambda\right]\right)$ pour $\mathrm{t}>\mathrm{t}_{\mathrm{o}}$

et

$$
\tau_{\mathrm{a}}=\tau_{\mathrm{a}_{0}} \text { pour } \mathrm{t}<\mathrm{t}_{\mathrm{o}}
$$

C'est finalement la valeur de chacun des paramètres $\tau_{a}$, $\Delta \tau_{\mathrm{a}}, \mathrm{t}_{\mathrm{o}}$ et $\lambda$ qui caractérise le collage d'un sédiment donné sur un type de surface donné.

Les valeurs expérimentales moyennes de ces différents paramètres concernant les essais réalisés sur la vase de l'étang de Salses-Leucate peuvent être déterminées comme suit :

- l'adhérence à temps de collage nul $\tau_{\mathrm{a}_{0}}=900 \mathrm{~Pa}$ (soit $0,45 \mathrm{cu}_{\mathrm{u}}$ )

- le temps minimum $t_{0}$ avant augmentation de l'adhérence : $t_{0}=10 \mathrm{~min}$

- la constante de temps $\lambda$ : elle est évaluée à $30 \mathrm{~min}$.

A la différence des essais en laboratoire, la valeur de la somme $\left(\tau_{\mathrm{a}_{0}}=\Delta \tau_{\mathrm{a}}\right)$ est pratiquement égale à la cohésion moyenne $c_{u}$ du sédiment.

L'accroissement de l'adhérence $\tau_{\mathrm{a}}=\mathrm{f}$ (temps) dans le cas du sédiment de l'étang de Salses-Leucate s'écrit sous la forme:

$$
\tau_{\mathrm{a}}=900+1100[1-\exp -(\mathrm{t}-10) / 30]
$$

Les variations de $\tau_{a}=f(t)$ pour les sédiments de la baie de Banyuls sont similaires à celles obtenues sur le site de Salses-Leucate (fig. 4). Cependant la comparaison entre ces deux études montre que:

- les valeurs mesurées, et en particulier celles de $\tau_{a \text { a }}$ sont nettement plus importantes :

$\tau_{\mathrm{a}_{0}}$ (adhérence à temps de collage nul) $=1250 \mathrm{~Pa}$.

La cohésion moyenne $c_{u}$ sur les sédiments provenant de ce site est plus élevée que pour les argiles de l'étang de Salses: $c_{u}=3500 \mathrm{~Pa}$. Cependant $\tau_{\mathrm{a}_{0}}=0,35 \mathrm{c}_{\mathrm{u}}$;

- l'adhérence augmente dès les premières minutes de collage : valeur très faible de $t_{0}(<1 \mathrm{~min})$;

- la constante de temps $\lambda$ est évaluée à 10 minutes.

Ainsi la modélisation des variations de l'adhérence $\tau_{a}$ en fonction du temps dans les conditions d'expérience en baie de Banyuls s'écrit :

$$
\tau_{a}=1250+2250[1-\exp -(t / 10)]
$$

Le tableau $n^{\circ} \|$ permet de résumer la comparaison entre les valeurs des paramètres déterminés pour chacun des deux sites et pour plusieurs stations expérimentales en précisant leurs principales caractéristiques géotechniques.

Les variations de $\tau_{a_{0}}$ correspondent aux accroissements de la cohésion du sédiment et corrélativement 
aux différences de teneurs en eaux. La constante de temps $\lambda$ qui traduit la rapidité d'augmentation de $\tau_{a}$ montre que l'influence de $t$ est nettement plus importante dans le cas des sédiments de la baie de Banyuls.

\subsection{Comparaison entre les essais en laboratoire et les essais in situ}

La différence essentielle existant entre les essais en laboratoire et les essais en nature réside dans le fait que les sédiments utilisés dans le premier cas sont totalement remaniés pour une granulométrie donnée. Ils ont perdu une partie de leurs caractéristiques géotechniques propres et présentent des cohésions plus faibles que dans la nature.

Cependant, le modèle proposé d'évolution de l'adhérence d'un sédiment sur une surface métallique en fonction du temps est identique dans les deux cas.

Si l'on considère les paramètres obtenus pour les plus fortes contraintes $\sigma_{n}$ pour les essais en laboratoire, le tableau comparatif $n^{\circ}$ III met en évidence à la fois le rôle des paramètres physiques (cohésion et teneur en eau) et l'influence du remaniement.

La valeur de la somme $\tau_{\mathrm{a}_{0}}+\Delta \tau_{\mathrm{a}}$ (adhérence à long terme sous forte contrainte) est pratiquement égale à la cohésion $c_{u}$, mais la valeur du rapport $\alpha=\tau_{a_{0}} / c_{u}$ varie nettement suivant qu'il s'agisse d'essais en laboratoire ou d'essais in situ pour tous les essais (fig. 5).

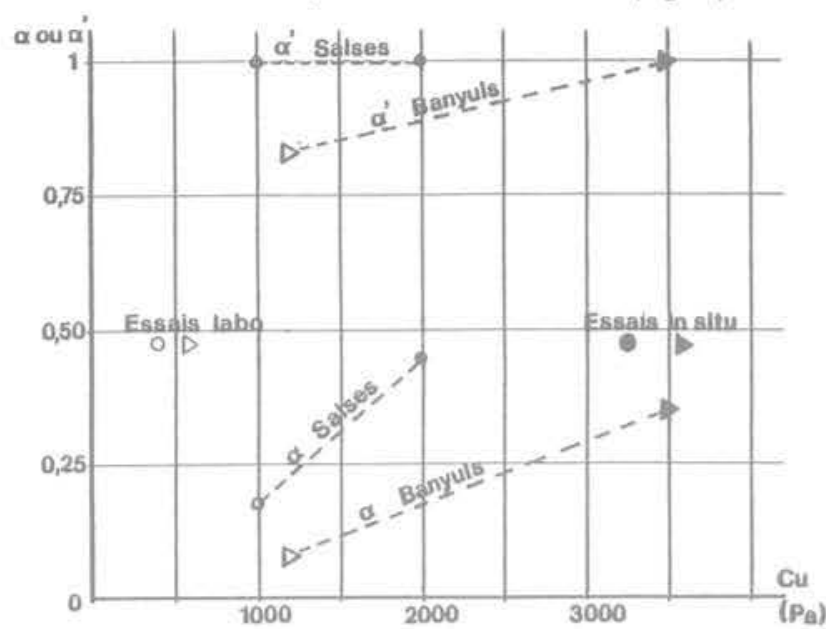

Fig. 5 Comparaison de $\alpha=f\left(c_{u}\right)$ et $\alpha^{\prime}=f\left(c_{u}\right)$ pour essais en laboratoire et essais in situ

\section{Conclusion}

Le temps est un facteur important de l'augmentation des forces d'adhérence d'un sédiment argileux au contact d'une surface métallique. Son influence semble prédominante dans les premières heures qui suivent ce contact. L'accroissement de l'adhérence $\tau_{\mathrm{a}}$ est surtout sensible au début du collage. $\tau_{\mathrm{a}}$ tend par la suite vers une limite proche de la valeur de cohésion $c_{u}$ du sédiment au-delà d'une période comprise entre 15 et 24 heures. Son influence est concrétisée par une équation de la forme:

$$
\tau_{\mathrm{a}}=\tau_{\mathrm{a}_{0}}+\Delta \tau_{\mathrm{a}}\left[1-\exp -\left(\mathrm{t}-\mathrm{t}_{\mathrm{o}}\right) \lambda\right]
$$

Le temps n'est qu'un facteur générateur de cette évolution. La nature minéralogique et les caractéristiques géotechniques du sédiment interviennent également pour définir les valeurs de l'adhérence.

La connaissance de l'évolution de $\tau_{\mathrm{a}}=\mathrm{f}(\mathrm{t})$, primordiale pour la modélisation de vis d'Archimède destinées à propulser un véhicule sous-marin, intervient dans le calcul de couple mécanique nécessaire au démarrage d'un engin et dans l'évaluation du rendement du système.

\section{Annexes}

Annexe $n^{\circ} 1$ - Mise en œuvre des essais en laboratoire :

Le principe des manipulations consiste à mesurer la force nécessaire à la traction d'une plaque posée horizontalement à la surface d'un sédiment reconstitué (fig. 6).

La force de traction est mesurée à partir d'un capteur de force et enregistrée avec une précision de $\pm 2 \%$.

Les plaques utilisées pour ces essais sont construites en tôle d'acier et permettent de choisir différents états de surface.

La section d'appui de ces plaques sur le sédiment est de :

$$
0,193 \mathrm{~m} \times 0,093 \mathrm{~m} \text { soit une surface de 0,018 } \mathrm{m}^{2} \text {. }
$$

La plaque posée sur de l'argile est tractée horizontalement par un système de tige-guide qui lui laisse un degré de liberté vertical lors de la transmission du mouvement.

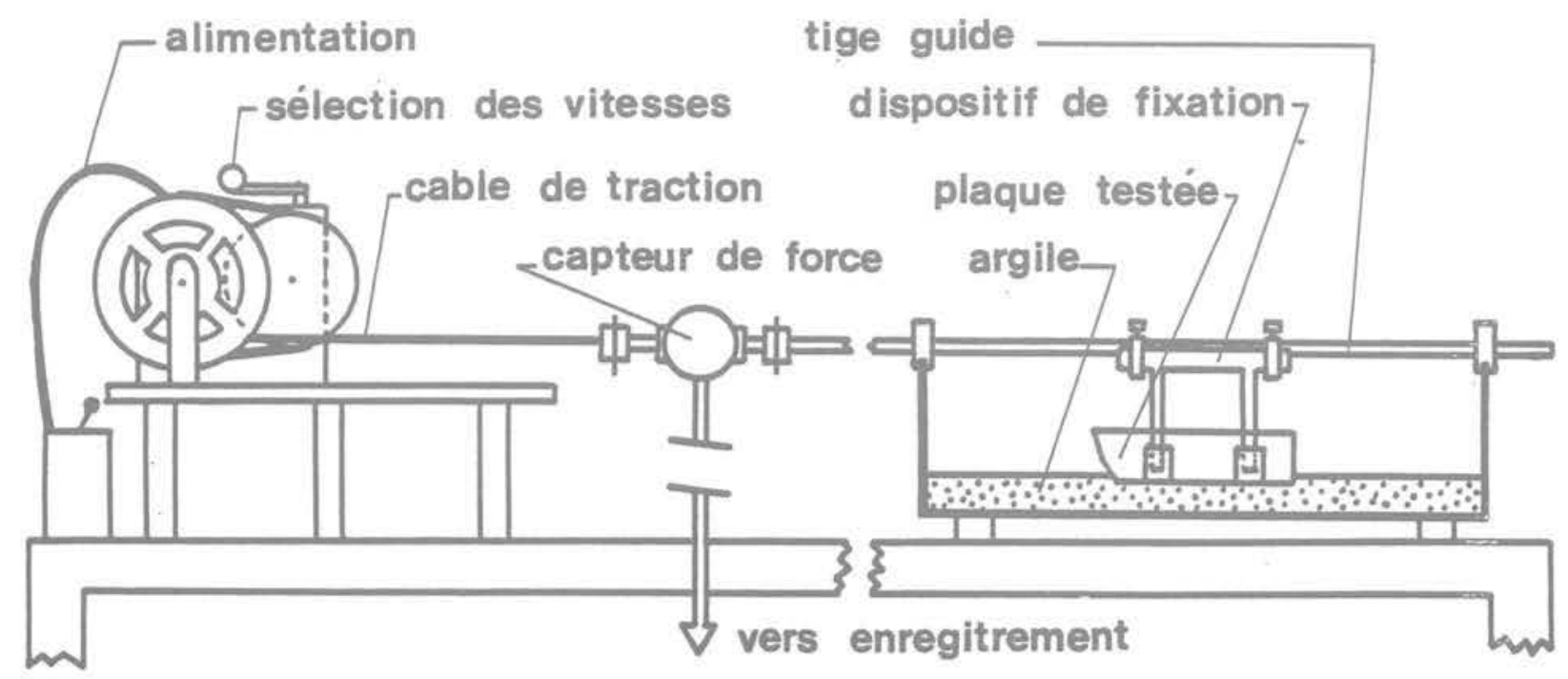

Fig. 6 Dispositif expérimental en laboratoire 
Le moteur de l'appareillage d'une boitte à cisaillement impose la vitesse de traction:

$$
v \text { traction }=1,21192 \cdot 10^{-3} \mathrm{~m} / \mathrm{mn} \text {. }
$$

Les sédiments utilisés sont contenus dans des bacs de $0,70 \mathrm{~m}$ de long, $0,25 \mathrm{~m}$ de large et $0,15 \mathrm{~m}$ de profondeur.

Les argiles préalablement malaxées dans l'eau sont réparties dans les bacs. II est alors nécessaire de respecter un temps de repos des sédiments de plusieurs jours pour obtenir une compaction et une cohésion compatibles avec les charges utilisées sans qu'il y ait un enfoncement notable des plaques.

\section{Caractéristiques des sédiments testés en cours de manipulation}

Vase grise de la baie de Banyuls:

Minéralogie : Illite dominante associée à la chlorite.

- Granulométrie : moyennement fin : $d_{50} \sim$ de 20 à $30 \mu \mathrm{m}$.

- Teneur en eau : $W \%$ de $120 \%$ (surface) à $50 \%$ $(-1 \mathrm{~cm})$.

- Densité humide apparente $\gamma_{\mathrm{h}}=$ de 1,42 (surface) à $1,71(-1 \mathrm{~cm})$.

- Cohésion $\mathrm{c}_{u}$ (FALL CONE) : de $500 \mathrm{~Pa}$ (surface) à $1200 \mathrm{~Pa}(-1 \mathrm{~cm})$.

- Limite de liquidité $W_{\mathrm{L}}=37,5$.

- Limite de plasticité $W_{\mathrm{P}}=28,6$.

- Indice de plasticité $I_{P}=8,7$.

Mélange: $75 \%$ vase grise de la baie de Banyuls $25 \%$ bentonite ( $\%$ en poids sec)

Teneur en eau W\%: de $130 \%$ (surface) à $68 \%$ $(-1 \mathrm{~cm})$.

Densité humidé apparente $\gamma_{\mathrm{h}}=$ de 1,40 (surface) à 1,60 $(-1 \mathrm{~cm})$.

Cohésion $c_{u}$ (FALL CONE) : $300 \mathrm{~Pa}$ (surface) à $700 \mathrm{~Pa}$ $(-1 \mathrm{~cm})$.

Limite de liquidité $W_{\mathrm{L}}=37,7$.

Limite de plasticité $W_{p}=28,8$,

Indice de plasticité $I_{p}=8,9$.

Kaolinite :

Teneur en eau $\mathrm{W} \%$ : de $106 \%$ (surface) à $42 \%$ $(-1 \mathrm{~cm})$.

Granulométrie : $0,7 \%$ de refus à $40, \mu \mathrm{m}$.

Densité humide apparente $\gamma_{\mathrm{h}}=$ de 1,43 (surface) à 1,75 $(-1 \mathrm{~cm})$.

Cohésion $c_{u}$ (FALL CONE) : de $5000 \mathrm{~Pa}$ (surface) à $6000 \mathrm{~Pa}(-1 \mathrm{~cm})$

Limite de liquidité $W_{L}=46$.

Limite de plasticité $W_{\mathrm{P}}=27$.

Indice de plasticité $I_{p}=19$.

Vase de l'étang de Salses-Leucate

Minéralogie: Illite prédominante avec kaolinite.

Granulométrie : $d_{50} \sim 30 \mu \mathrm{m}$.

Teneur en eau $\mathrm{W} \%$ : $61 \%$ à $77 \%$.

Densité humide apparente $\gamma_{h}: 1,57$ à 1,62.

Cohésion $c_{u}$ (FALL CONE) : $1000 \mathrm{~Pa}$.

Limite de liquidité $W_{\mathrm{L}}=50$.

Limite de plasticité $W_{P}=37$.

Indice de plasticité $I_{p}=13$.

\section{Annexe $\mathrm{n}^{\circ} 2$ - Mise en œuvre des essais in situ}

Le principe de ces manipulations consiste à mesurer les efforts nécessaires à l'enfoncement et à l'arrachement de plaques en tôle de très faible épaisseur dans les vases marines (fig. 7).

Une structure de protection sur le fond marin permet de guider verticalement les plaques et de les garantir des mouvements hydrodynamiques en cours de manipulation.

Les plaques utilisées en tôle mince de $0,003 \mathrm{~m}$ d'épaisseur, ont quatre sections différentes:

$$
\begin{aligned}
& \text { hauteur: } 1,10 \mathrm{~m} \text {; longueur : } 0,40 \mathrm{~m} \\
& \text { hauteur : } 1,00 \mathrm{~m} \text {; largeur : } 0,60 \mathrm{~m} \\
& \text { hauteur: } 1,00 \mathrm{~m} \text {; largeur : } 0,80 \mathrm{~m} \\
& \text { hauteur : } 1,00 \mathrm{~m} \text {; largeur : } 1,00 \mathrm{~m}
\end{aligned}
$$

Une plaque posée verticalement sur le fond marin s'enfonce rapidement sous l'effet de son propre poids jusqu'à stabilisation.

La résistance à l'enfoncement est pratiquement due aux seuis frottements exercés par le sédiment sur les surfaces en contact de la plaque : en effet, compte tenu des caractéristiques du sédiment (faible cohésion) de la très faible épaisseur de la tôle, la résistance par effet de pointe sous la plaque peut raisonnablement être négligée.

Nous déduisons ainsi une valeur initiale de frottement unitaire immédiatement après enfoncement.

La force de collage " $G$ n à l'arrachement au bout d'un temps " $t$ " s'exprime ainsi

$$
\mathrm{G}=\mathrm{F}_{\mathrm{T}}-\mathrm{P} \text { où }
$$

$P$ est le poids déjaugé de la plaque et $F_{\mathrm{T}}$ la force de traction mesurée au dynamomètre.

L'adhérence est la force $T_{F}$ par unité de surface exercée sur les deux faces de la plaque.

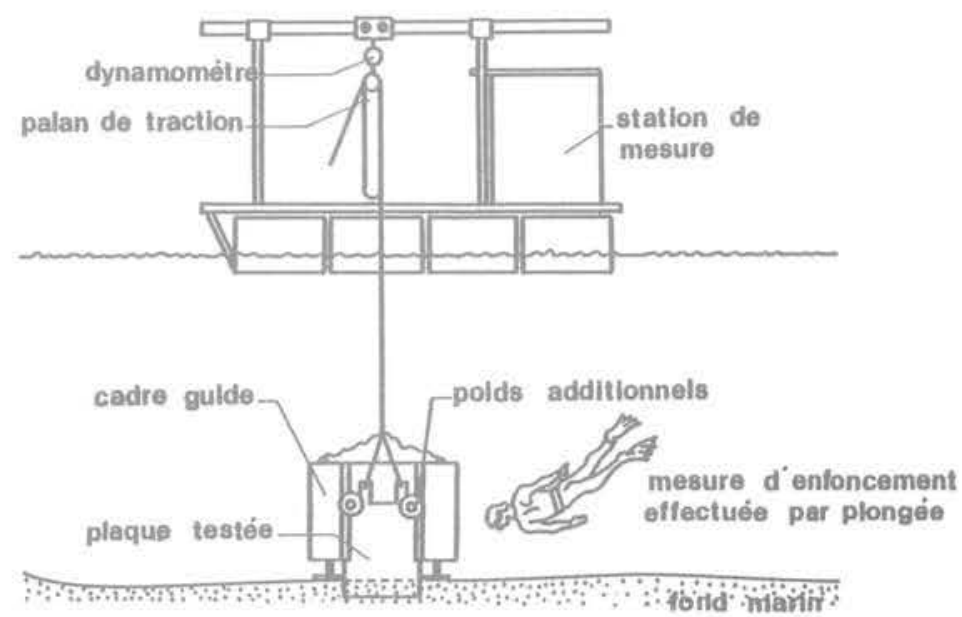

Fig. 7 Dispositif expérimental in situ

\section{Caractéristiques des sédiments marins}

Vase de l'étang de Salses-Leucate/Station de mesure ES-5

Minéralogie : llite dominante avec kaolinite.

Granulométrie : sédiment fin moyennement classé médiane $\mathrm{d}_{50}=$ de 4 à $60 \mu \mathrm{m}$,

Sorting index $S_{0}=3,3$ à 10 , 
Teneur en eau W \% : $80 \%$.

Cohésion (FALL CONE TEST) $c_{u}=2000 \mathrm{~Pa}$.

Indice de compression $\mathrm{C}_{0}=0,37$,

Vase de la baie de Banyuls

Minéralogie : Illite dominante associée à la chlorite.

Granulométrie : médiane $\mathrm{d}_{50}=$ de 20 à $30 \mu \mathrm{m}$.

Sorting index $S_{0}=1,3$ à 4

(avec nombreux débris coquilliers)

Teneur en eau $\mathrm{W} \%$ : station $\mathrm{B} 15 \mathrm{~W} \%=37 \%$, station $B 21 \mathrm{~W} \%=39 \%$.

Cohésion (FALL CONE TEST): station B15 $\mathrm{c}_{u}=$ $34000 \mathrm{~Pa}$; station $\mathrm{B} 21 \mathrm{c}_{4}=3000 \mathrm{~Pa}$.

Indice de compression $\mathrm{C}_{c}$ : station $\mathrm{B} 15 \mathrm{C}_{\mathrm{c}}=0,33$; station $\mathrm{B} 21 \mathrm{C}_{\mathrm{c}}=0,17$.

\section{Bibliographie}

[1] J.-Y. Boisson (1981). Étude de l'adhérence de sédiments argileux à des surfaces métalliques. Thèse présentée à l'Université de Toulouse, soutenue le 7 février 1981 pour obtenir le grade de Docteur de $3^{\circ}$ cycle.

[2] A. Caquot et J. Kerisel (1966). Traité de mécanique des sols. Gauthiers Villars, Paris.

[3] J. Costet et G. Sanglerat (1975), Cours pratique de mécanique des sols. Dunod, Paris, tomes 1 et 2.

[4] T.R. Chari, S.N. Guha, K. Muthukrishnaiah (1978). Adhesive resistance of under consolidated sediments. Faculty of Engineering and Applied Science, Memorial University of Newfoundland, St John's Canada.

[5] R.A. Erchul et R.J. Smith (1969). Lubricant and polymer reduction of sediment adhesion, décembre 1969. Proceeding ASCE Conference, Civil Engineering in the Oceans II, pages $621-640$.
[6] J.I. Fuller (1975). Behavior of Mechanical elements in submerged clays of low shear strengh. Kennecott Exploration Inc. Paper Number OTC 2242.

[7] J. Lee Mona (1973). Breakout of partially embedded objects from cohesive sea floor soils. Offshore Technology Conference. Paper Number OTC 1904.

[8] G.G. Meyerhof (1961). Some problems in the desing of rigid retaining walls. Proceedings, 15 th Canadian. Soils mechanics, Conference Ottawa, p. 59-79.

[9] G.G. Meyerhof and J.I. Adams (1968). The ultimate uplift capacity of foundations. Canadian geotechnical Journal, vol. V, $n^{\circ} 4$, nov., pp. 225-244.

[10] Dirk Neuhaus (1973). Étude expérimentale d'un modèle de pieu dans un sol cohérent. Thèse $3^{\circ}$ cycle, Université de Grenoble.

[11] J.G. Potyondy (1961). Skin friction between cohesive granular soils and construction material. Géotechnique, Tome II, $n^{\circ} 4$, pp. 339-353.

[12] G. Sanglerat (1977). Le pénétromètre statiquedynamique et ses diverses applications pratiques. Conférence.

[13] J.M. Tomlinson (1957). The adhesion of piles in clay soils. Proceedings 4 th Int. Conf Soil Mechanic 2, pp. 66-71, London.

[14] J.M. Tomlinson (1969). Fondation design and construction. $2^{\circ}$ édition, 765 pages, I. Pitman and Son Ltd London.

[15] S.A. (1971). Breakout resistance of objects embedded in ocean bottom. Journal of Soil mechanics and fondations division. Proceeding of the American Society of Civil Engineers. Septembre 1971, pp. 11831205 .

[16] M.C. Wang, K.R. Demars, V.A. Nacci (1977). Breakout capacity of model succion anchors in soil. Canadian geotechnic journal $n^{\circ} 14$, pp. 246-257. 